\title{
Combined drug therapy for management of jetlag
}

\begin{abstract}
Current therapies for jet lag include melatonin, timed light exposure and modification of sleep schedule. There has not been a study looking at combination therapy to improve both daytime sleepiness and reduce sleep disturbance associated with jet lag. We describe a case where combined drug therapy with modafinil and zolpidem was effective in treating jetlag in a business traveler with 8 hour time zone change.
\end{abstract}

Volume 3 Issue 5 - 2016

\author{
Andres Endara Bravo,' Adam Anglyn ${ }^{2}$ \\ 'Southeastern Pulmonary and Sleep Clinic, USA \\ ${ }^{2}$ Department of Medical Education, USA
}

\author{
Correspondence: Andres Endara Bravo, Southeastern \\ Pulmonary and Sleep Clinic, 40I West 27th Street, Lumberton, \\ NC 28358, Tel 910-674-5198, Fax 910-272-7155, \\ Email asendara@yahoo.com
}

Received: July 15, 2016 | Published: September 01, 2016

\section{Report of case}

A 38-year-old professional man without any medical history, presented to the sleep clinic prior to a one week business trip from United States to United Arab Emirates. Patient requested medical therapy to help him adjust and perform during his trip in view of 8-hour time zone change. The patient had tried melatonin, timed light exposure and sleep schedule modification on prior occasions without prompt results. Patient was prescribed modafinil 200mg in the morning and zolpidem $10 \mathrm{mg}$ at bedtime for the duration of his trip. Upon return to the clinic, patient reported good vigilance during the day and average sleep time of 7hours at night while abroad. The patient reported no adverse effects to the medications. Upon return to the United States, patient discontinued the combined therapy and returned to his usual schedule without major disturbances.

\section{Discussion}

Current therapies for jet lag include timed melatonin, timed light exposure, and modification of sleep schedule. The circadian rhythm adjusts in travelers up to one time zone per day for eastward travel and 1.5 time zones per day for westward travel. ${ }^{1}$ Eastward travel requires persons to advance their circadian rhythm. Current therapy modalities are recommended when traveling more than 6time zones eastward or 12 time zones westward.

The use of armodafinil $150 \mathrm{mg}$ has been reported in 427 participants with jet lag disorder after 6hour time zone change. ${ }^{2}$ Participants reported near normal daytime sleepiness scores with a
MSLT $>10$ minutes compared to $<5$ minutes in the placebo group. In another study, zolpidem $10 \mathrm{mg}$ was associated with reduced sleep disturbance in patients with jetlag with an increase in total sleep time and a decrease in number of awakenings per night. ${ }^{3}$

The combination therapy of modafinil and zolpidem has not been previously studied/reported. In our patient, the short term use of combination therapy with modafinil $200 \mathrm{mg}$ in the morning and zolpidem 10mg at bedtime was associated with Epworth sleepiness score less than 10 and average total sleep time of 7hours.

\section{Acknowledgements}

None.

\section{Conflict of interest}

The author declares no conflict $\mathrm{f}$ interest.

\section{References}

1. Aschoff J, Hoffmann K, Pohl H, et al. Re-entrainment of circadian rhythms after phase-shifts of the Zeitgeber. Chronobiologia. 1975;2(1):23-78.

2. Rosenberg RP, Bogan RK, Tiller JM, et al. A phase 3, double-blind, randomized, placebo-controlled study of armodafinil for excessive sleepiness associated with jet lag disorder. Mayo Clin Proc. 2010;85(7):630638.

3. Jamieson AO, Zammit GK, Rosenberg RS, et al. Zolpidem reduces the sleep disturbance of jet lag. Sleep Med. 2001;2(5):423-430. 